\title{
Differential Encoding of Predator Fear in the Ventromedial Hypothalamus and Periaqueductal Grey
}

\author{
Maria Esteban Masferrer, ${ }^{1}{ }^{\circledR}$ Bianca A. Silva, ${ }^{1}$ Kensaku Nomoto, ${ }^{2}$ Susana Q. Lima, ${ }^{2}$ and Cornelius T. Gross ${ }^{1}$ \\ ${ }^{1}$ Epigenetics and Neurobiology Unit, EMBL Rome, European Molecular Biology Laboratory, Monterotondo RM 00015, Italy, and ${ }^{2}$ Champalimaud \\ Centre for the Unknown, Lisbon 1400-038, Portugal
}

The ventromedial hypothalamus is a central node of the mammalian predator defense network. Stimulation of this structure in rodents and primates elicits abrupt defensive responses, including flight, freezing, sympathetic activation, and panic, while inhibition reduces defensive responses to predators. The major efferent target of the ventromedial hypothalamus is the dorsal periaqueductal gray (dPAG), and stimulation of this structure also elicits flight, freezing, and sympathetic activation. However, reversible inhibition experiments suggest that the ventromedial hypothalamus and periaqueductal gray play distinct roles in the control of defensive behavior, with the former proposed to encode an internal state necessary for the motivation of defensive responses, while the latter serves as a motor pattern initiator. Here, we used electrophysiological recordings of single units in behaving male mice exposed to a rat to investigate the encoding of predator fear in the dorsomedial division of the ventromedial hypothalamus (VMHdm) and the dPAG. Distinct correlates of threat intensity and motor responses were found in both structures, suggesting a distributed encoding of sensory and motor features in the medial hypothalamic-brainstem instinctive network.

Key words: defensive behavior; electrophysiology; optogenetics; periaqueductal gray; predator fear; ventromedial hypothalamus

\section{Significance Statement}

Although behavioral responses to predatory threat are essential for survival, the underlying neuronal circuits remain undefined. Using single unit in vivo electrophysiological recordings in mice, we have identified neuronal populations in the medial hypothalamus and brainstem that encode defensive responses to a rat predator. We found that both structures encode both sensory as well as motor aspects of the behavior although with different kinetics. Our findings provide a framework for understanding how innate sensory cues are processed to elicit adaptive behavioral responses to threat and will help to identify targets for the pharmacological modulation of related pathologic behaviors.

\section{Introduction}

Electrical stimulation and lesion studies identified the medial hypothalamus as a central organizer of innate goal-directed behaviors associated with defense and reproduction (Malsbury et al., 1977; Kruk et al., 1979; Pfaff and Sakuma, 1979a,b; Canteras

Received Mar. 22, 2018; revised Aug. 20, 2020; accepted 0ct. 21, 2020.

Author contributions: M.E.M. and C.T.G. designed research; M.E.M., B.A.S., and K.N. performed research; K.N. and S.Q.L. contributed unpublished reagents/analytic tools; M.E.M. analyzed data; M.E.M. and C.T.G. wrote the paper.

This work was supported by The European Molecular Biology Laboratory and the European Research Council Advanced Grant COREFEAR (to C.T.G.). We thank Francesca Zonfrillo, Roberto Voci, Matteo Gaetani, Monica Serra, and Valerio Rossi for mouse husbandry; the EMBL Histology and Microscopy Facilities; and Angelo Raggioli for laboratory management support.

The authors declare no competing financial interests.

Correspondence should be addressed to Cornelius T. Gross at gross@embl.it.

https://doi.org/10.1523/JNEUROSCI.0761-18.2020

Copyright $\odot 2020$ Esteban Masferrer et al.

This is an open-access article distributed under the terms of the Creative Commons Attribution 4.0 International license, which permits unrestricted use, distribution and reproduction in any medium provided that the original work is properly attributed. et al., 1997; Canteras, 2002; Lin et al., 2011; Yang et al., 2013; Silva et al., 2013). Evidence for its anatomic and molecular conservation across vertebrates (Braak and Braak, 1992; Koutcherov et al., 2002; Kurrasch et al., 2007) suggests that it forms an ancient control center for survival responses that may date back as far as the evolution of bilaterians (Tessmar-Raible et al., 2007; Arendt et al., 2016). Anatomical tract-tracing and cFos mapping studies in rats exposed to cats identified a set of three interconnected medial hypothalamic nuclei, the anterior hypothalamic nucleus (AHN), dorsomedial division of the ventromedial hypothalamus (VMHdm), and dorsal premammillary nucleus (PMD), which together comprise the medial hypothalamic defensive system (Canteras, 2002). The VMHdm occupies a central position in the network because it receives direct input from olfactory and pheremonal sensory processing areas in the cortical and medial amygdala (Swanson and Petrovich, 1998; Choi et al., 2005; Bergan et al., 2014) necessary to detect threat-related stimuli and at the same time provides direct outputs to the dorsal periaqueductal gray (dPAG), 
considered to be the behavioral and autonomic motor pattern initiator for defensive responses (Blanchard et al., 1981; Bandler and McCulloch, 1984; Fuchs et al., 1985; Graeff, 1994; Swanson, 2000; Canteras, 2002).

Recent work in mice has begun to dissect the medial hypothalamic defensive system at the circuit level. Optogenetic stimulation of neurons in VMHdm (Lin et al., 2011; Kunwar et al., 2015) or their projections to AHN or PAG (Wang et al., 2015) elicited freezing and flight behavior and pharmacogenetic inhibition or genetic ablation of neurotransmission in VMHdm reduced defensive responses to predators (Silva et al., 2013; Kunwar et al., 2015). Neurons in VMHdm appear to selectively support defensive behaviors elicited by predators, as defensive responses to a conspecific were unaffected by pharmacogenetic inhibition (Silva et al., 2013; but see Kunwar et al., 2015). Similarly, optogenetic stimulation of neurons in dPAG elicited freezing and flight (Deng et al., 2016; Tovote et al., 2016; Evans et al., 2018) and pharmacogenetic inhibition reduced defensive responses to predator (Silva et al., 2013). However, pharmacogenetic inhibition of dPAG did not impair the acquisition of a fear memory formed by exposure to the predator, while VMHdm inhibition did (Silva et al., 2016b), suggesting a role for PAG limited to the expression of defensive behaviors and a broader role for $\mathrm{VMH}$ in encoding an internal, motivational state required both for the expression and memory of predator defense (Silva et al., 2016a,b). Data from deep brain electrical stimulation studies in humans appear to support this distinction, with stimulation of VMHdm eliciting feelings of dread, impending doom, and panic (Wilent et al., 2010) and stimulation of dPAG eliciting sensations of being chased (Amano et al., 1982).

A potential role for $\mathrm{VMHdm}$ in encoding defensive motivation is also supported by analogy with single unit electrophysiological recordings and calcium imaging in the ventrolateral division of the VMH (VMHvl), a part of the medial hypothalamic reproductive system, in male mice exhibiting aggression toward male intruders (Lin et al., 2011; Falkner et al., 2014, 2016; Remedios et al., 2017; Krzywkowski et al., 2020). In these studies, VMHvl neurons showed increased firing when exposed to awake males, anesthetized males, or male urine, and the intensity of activation was correlated with the latency and duration of future attacks, demonstrating the encoding of both sensory as well as motivational features of the behavior. The capacity of neural activity in VMHvl to motivate attack behavior was subsequently tested by showing that optogenetic stimulation of VMHvl could drive lever pressing behavior to get access to an intruder (Falkner et al., 2016). Single unit recordings in the VMHdm of behaving animals have not been reported, but by analogy with VMHvl such neural activity should similarly scale with both sensory features of the threat as well as the probability or intensity of the defensive response.

In $\mathrm{APAG}$, on the other hand, electrophysiological recordings of single units in awake behaving mice exposed to a rat identified two major classes of neurons: flight cells, whose firing increased during flight from the predator, and assessment cells, whose firing increased with decreasing distance from the predator (Deng et al., 2016). These findings demonstrated that dPAG neuron activity encodes not only defensive motor actions (flight) but also sensory aspects of threat distance and intensity (assessment). Together, these findings suggest that a simple model in which VMHdm firing encodes threat intensity and dPAG neurons are triggered to produce defensive behaviors when this activity reaches a given threshold is likely to be incorrect. Here, we investigated single unit activity in both VMHdm and $\mathrm{VPAG}$ in awake behaving mice exposed to a rat to understand whether and how predator defense behavior is differentially encoded in these structures. Our data revealed that both VMHdm and dPAG contain assessment and flight cells, suggesting a distributed encoding of sensory and motor aspects of defense across these structures. However, correlations between defensive behavior and firing rates were different in VMHdm and $\mathrm{PPAG}$, confirming a hierarchical encoding of defense between medial hypothalamus and brainstem that incorporates brainstem-hypothalamus feedback control.

\section{Materials and Methods}

Animals

Adult C57BL/6 $\mathrm{N}$ male mice were used for single units recording in VMHdm (experiment $2, N=4$; experiment $3, N=6)$ and dPAG $(N=8)$. For recording in VMHdm and simultaneous optogenetic and pharmacogenetic manipulation of dPAG, vesicular glutamate transporter 2 Creexpressing male mice ( $N=4$, Vglut2::Cre; Borgius et al., 2010) were used. Adult male SHR/NHsd rats (Harlan) were used as predators. All experimental mice were singly housed after the surgery at $22-25^{\circ} \mathrm{C}$ under a $12 / 12 \mathrm{~h}$ light/dark cycle with water and food ad libitum. All animals were handled according to protocols approved by the Italian Ministry of Health (541/2015-PR).

\section{Surgery}

Surgeries were performed in 8- to 10 -week-old mice, under deep anesthesia (isoflurane $3 \%$ for induction and $1.5-2 \%$ in $\mathrm{O}_{2}$ for maintenance). Stereotaxic surgeries were performed using RWD Life Sciences/Kopf Instruments frames. For extracellular recordings, mice were implanted with a commercial movable bundle with 16 insulated tungsten microwires bared tip $(23 \mu \mathrm{m}$ in diameter, impedance $\sim 600 \mathrm{k} \Omega)$ plus a $300-\mu \mathrm{m}$ diameter silver wire to be used as ground (Innovative Neurophysiology; Nomoto and Lima, 2015) into the VMHdm (-0.95 $\mathrm{mm}$ posterior to bregma, $+0.3 \mathrm{~mm}$ lateral, and $-5.35 \mathrm{~mm}$ ventral to the brain surface) or $\mathrm{dPAG}(-4.1 \mathrm{~mm}$ posterior to bregma, $+1.18 \mathrm{~mm}$ lateral, and -2.36 $\mathrm{mm}$ ventral to the skull surface, at $26^{\circ}$ lateral angle). For the dPAG optogenetic activation and DREADD chemogenetic inhibition, mice were injected with a 1:1 $\operatorname{mix}(0.3 \mu \mathrm{l}, 0.05 \mu \mathrm{l} / \mathrm{min})$ of AAV5-Ef 1a:: DIOhChR2(E123T/T159C)- EYFP and AAV2-hSyn:: DIO-hM4D- Cherry viruses (Addgene) ipsilateral to the recording site $(-4.1 \mathrm{~mm}$ posterior to bregma, $-2.36 \mathrm{~mm}$ ventral to the skull surface, at $26^{\circ}$ lateral angle). Following viral infection, an optic fiber (0.22 numerical aperture, $225-\mu \mathrm{m}$ core diameter, ceramic ferrule: $1.25-\mathrm{mm}$ outer diameter, Thorlabs) was implanted above the dPAG $(-4.1 \mathrm{~mm}$ posterior to bregma, $+1.18 \mathrm{~mm}$ lateral, $-2.25 \mathrm{~mm}$ ventral to the skull surface, at $26^{\circ}$ lateral angle). Two stainless steel screws (RWD Life Science) were fixed permanently into the posterior and anterior portions of the skull to serve as ground and fixation. Implants were fixed on the skull using dental acrylic (DuraLay Reliance). After surgery mice were injected with carprofen (Rimadyl, $5 \mathrm{mg} / \mathrm{kg}$, s.c.) for $3 \mathrm{~d}$ to control pain and inflammation. All animals were allowed to recover for at least two weeks before testing.

\section{Behavioral assay}

The experimental apparatus (adapted from Silva et al., 2013) was made of transparent Plexiglas and composed of a stimulus chamber $(25 \times 25 \times$ $25 \mathrm{~cm})$ connected by an opening $(2 \times 25 \mathrm{~cm})$ with a removable door to a narrow corridor $(12.5 \times 60 \times 30 \mathrm{~cm})$. At the beginning of each session the experimental animal was connected to the acquisition system and monitored for $20 \mathrm{~min}$ in the home cage to confirm signal stability before being placed into the experimental apparatus. Each session consisted of $10 \mathrm{~min}$ of habituation in which the door was opened to allow the animal to freely explore the corridor and stimulus chamber, followed by closing the door while the animal was in the corridor and placement of the rat in the stimulus compartment. Exploratory contact between the rat and the subject was permitted for $10 \mathrm{~min}$. Subsequently, the rat was removed and the door opened, and the mouse was allowed to explore the corridor and stimulus chamber for another $5 \mathrm{~min}$. At the end of each session the electrodes were advanced $60 \mu \mathrm{m}$ and mice checked 
again 1-2 d later. Videos were recorded at 30 frames/s to extract position and velocity of the animal (CinePlex Studio, Plexon). Video and neural data were synchronized offline and the behavior was manually scored (CinePlex Editor, Plexon).

\section{Optogenetic activation and DREADD inhibition}

Experimental subjects injected with a 1:1 mixture of ChR2 and hM4D expressing viruses were injected intraperitoneally with saline before starting the test and with the hM4D receptor activator clozapine-N-oxide $(\mathrm{CNO} ; 3 \mathrm{mg} / \mathrm{Kg}$, i.p.) shortly after the initial post-rat phase, and $1 \mathrm{~h}$ later, the whole behavioral assay was repeated. This group of mice was stimulated using blue light $(465 \mathrm{~nm})$ from a LED source via high performance patch cables (Plexbright, Plexon). Five trains of pulses of high frequency ipsilateral stimulation $[20 \mathrm{~Hz}, 15-\mathrm{ms}$ pulses, 9-12 MW, $1 \mathrm{~min}$ inter train interval] was used to stimulate ChR2-expressing cells in $\mathrm{dPAG}$ in the home cage shortly after the saline injection and repeated $1 \mathrm{~h}$ after the administration of $\mathrm{CNO}$.

\section{Electrophysiology}

During the recording sessions mice were connected to a lightweight head stage (1.03 g, gain $20 \times$, Plexon). The head stage was connected to a 16-channel analog amplifier (gain $50 \times$, Plexon) and neural activity was checked online. When needed, one of the 16 wires was used as a common reference for the other channels. If at least one unit was identified, the behavioral paradigm was started. Otherwise, the bundle of electrodes was re-adjusted by moving the screw to advance it by $60 \mu \mathrm{m}$ followed by a waiting period of $24 \mathrm{~h}$ before the next recording session. The neural signal was acquired (digitized at $40 \mathrm{kHz}$ ) and filtered to separate high frequency $(300 \mathrm{~Hz}$ to $8 \mathrm{kHz}$ ) and low frequencies (100-1800) using a Neural Data Acquisition System (Omniplex, Plexon). Information was stored for offline analysis. Spikes were sorted offline from the high frequency filtered data (Offline Sorter, Plexon) based on 3D principal component analysis (Extended Data Figs. 2-1, 3-1). Unit isolation was verified using autocorrelation histograms, and cross-correlation histograms were used to detect units appearing in more than one channel. Three criteria were used to consider the recorded cell as a single unit: (1) signal-to noise ratio $>3 \sigma$, (2) stable waveform shape during the recording, and (3) percentage of spikes occurring with ISIs $<2 \mathrm{~ms}$ must be $<0.1 \%$ (Extended Data Figs. 2-1, 3-1). Low-frequency filtered data were used to analyze the amplitude of the first evoked responses generated in dmVMH optogenetic trains stimuli applied in $\mathrm{dPAG}$.

\section{Physiology analysis}

Confirmation of well-isolated single units through auto and crosscorrelograms and Z-score calculation for each neuron/recording session were performed using NeuroExplorer software Convined peristimulus time histograms (PSTHs) and peristimulus rasters were computed using MLIB-toolbox scripts (Stüttgen, 2020) for analyzing spike data (MATLAB, MathWorks). For each neuron, a mean spike density function was constructed by applying a Gaussian kernel $(\sigma=10 \mathrm{~ms})$. Peristimulus graphs were calculated using 10-ms bins. For the mean PSTH, the firing rate for each unit was averaged across trials/mice.

\section{Statistics}

To define units as "assessment" or "flight" cells, Wilcoxon signed-rank test was used where activity 2-s pre-flight or post-flight onset was compared 20 times with a 2-s randomly chosen baseline time window. In order to avoid a phase effect in the firing rate, only data from the corresponding phase (pre-rat, rat, or post-rat) were used to calculate the baseline. Cells showing significant change during flight or assessment phases were classified consequently (MATLAB signrank function, $p<0.001$ ). To confirm significance in the VMHdm-evoked response changes as consequence of optogenetically activation of dPAG GABAergic cells before and after being chemogenetically blocked, paired $t$ test was calculated using Prism 7 (GraphPad; mice $N=7$ sessions $N=14$ )

Histology

At the end of the experiment, a small electrolytic lesion was made around the tip of the electrode $(20 \mu \mathrm{A}, 40 \mathrm{~s}$; Cibertec stimulator). Serial coronal sections $(40 \mu \mathrm{m})$ of perfused brains $(1 \times$ PBS and $4 \%$ PFA in $1 \times$
$\mathrm{PB})$ were cut on a cryomicrotome, stained using Nissl technique $(0.1 \%$ Cresyl Violet), and visualized under the microscope to verify the electrode placement. For tracking final fiber placement, brain slices were stained by adding DAPI $(5 \mathrm{mg} / \mathrm{ml})$ to the mounting medium (MOWIOL). Pictures were taken with a fluorescence microscope (Leica) using a $10 \times$ objective to check the viral infection.

\section{Results}

Current approaches for understanding the encoding of behavior in the firing of single neurons involve recording single unit electrical activity in awake behaving animals showing robust and repeated responses to an eliciting stimulus over multiple sessions and days. To achieve such conditions we modified our existing mouse predator defense test (Silva et al., 2013) so that the mouse could be transferred each day from its home cage to the testing apparatus consisting of a $60-\mathrm{cm}$-long corridor connected to a larger $25 \times 25 \mathrm{~cm}$ stimulus chamber via a small opening (Fig. $1 A)$. At the beginning of each session the electrode connector of the experimental animal was plugged into the recording cable and the animal was returned to its home cage for $20 \mathrm{~min}$ to stabilize the recording. At the start of the experiment the animal was transferred to the experimental apparatus and allowed to explore during a 10-min habituation phase. At a moment when the experimental animal was in the corridor compartment a rat was placed into the stimulus chamber and the defensive behavior of the mouse was observed for a further $10 \mathrm{~min}$ (rat phase). At the end of the session, the rat was removed and the mouse was allowed to explore for an additional $5 \mathrm{~min}$ (post-rat phase). Quantification of the cumulative dwell time in the apparatus revealed a clear decrease in time spent near the stimulus chamber in the presence of the rat (Fig. $1 B, C$ ). Notably, in the presence of the rat the mouse repeatedly conducted a sequence of behaviors in which it moved cautiously toward the stimulus chamber (assessment) and then turned to initiate a rapid movement (flight) away from the rat (Fig. 1D). Robust assessment-flight sequences were seen over multiple rat phase testing sessions (9.3 \pm 0.5 per session; $7 \pm 2$ sessions per animal, maximum one session per day, $N=12$ ). Although approach behavior was frequent also during the habituation and post-rat phases, under these conditions, no flights were observed (habituation $0 / 330$, rat $324 / 334$, post-rat 0/163). Animals were implanted with a 16microwire electrode fitted to a movable drive into either the VMHdm or dPAG (Figs. 2A, 3A) and allowed to recover for at least two weeks before testing. Electrodes were advanced at the end of each session and putative single units identified before the start of testing using standard spike sorting methods. A total of 371 putative single units recorded in 13 mice across a total of 101 sessions satisfied all criteria and were included in the final analysis. To identify units whose firing pattern was correlated with approach-flight behavior we aligned the firing of units to the start of each flight event across sessions as measured by an overhead video tracking apparatus and assessed correlations statistically.

In $\mathrm{dPAG}, 43 \%$ of single units (30/69) were significantly correlated with approach-flight behavior (Fig. 2B). The patterns of correlation could be clustered into three types: cells that increased firing during approach ("assessment+" cells, 13\%, 9/69; Fig. 2C; Extended Data Fig. 2-2B; Movie 1), cells that decreased firing during approach ("assessment-" cells, 12\%, 8/69; Fig. 2D; Extended Data Fig. 2-2B; Movie 2), and cells that increased firing during flight (flight cells, 16\%, 11/69; Fig. 2E; Extended Data Fig. 2-2B; Movie 3). One single unit consistently decreased firing during flight and another one was correlated 
A

Pre-rat

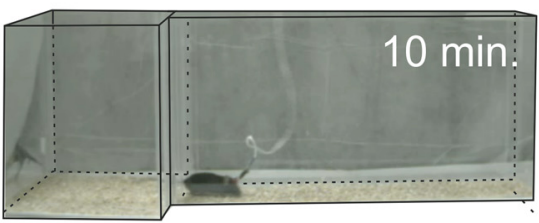

B

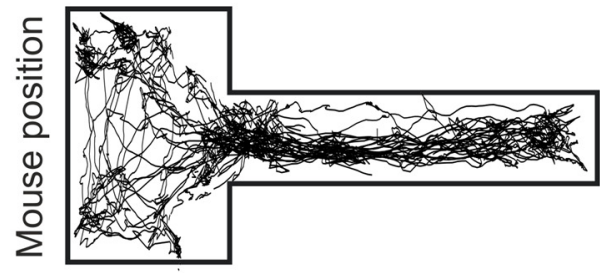

Rat

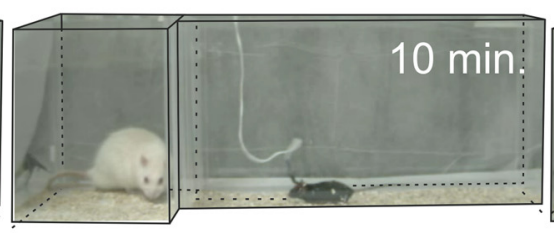

Post-rat

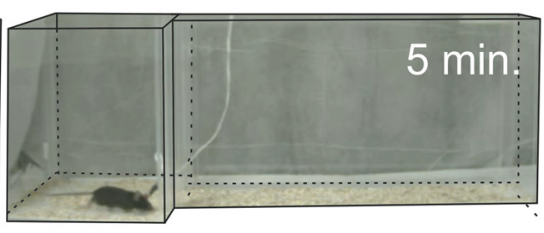

C
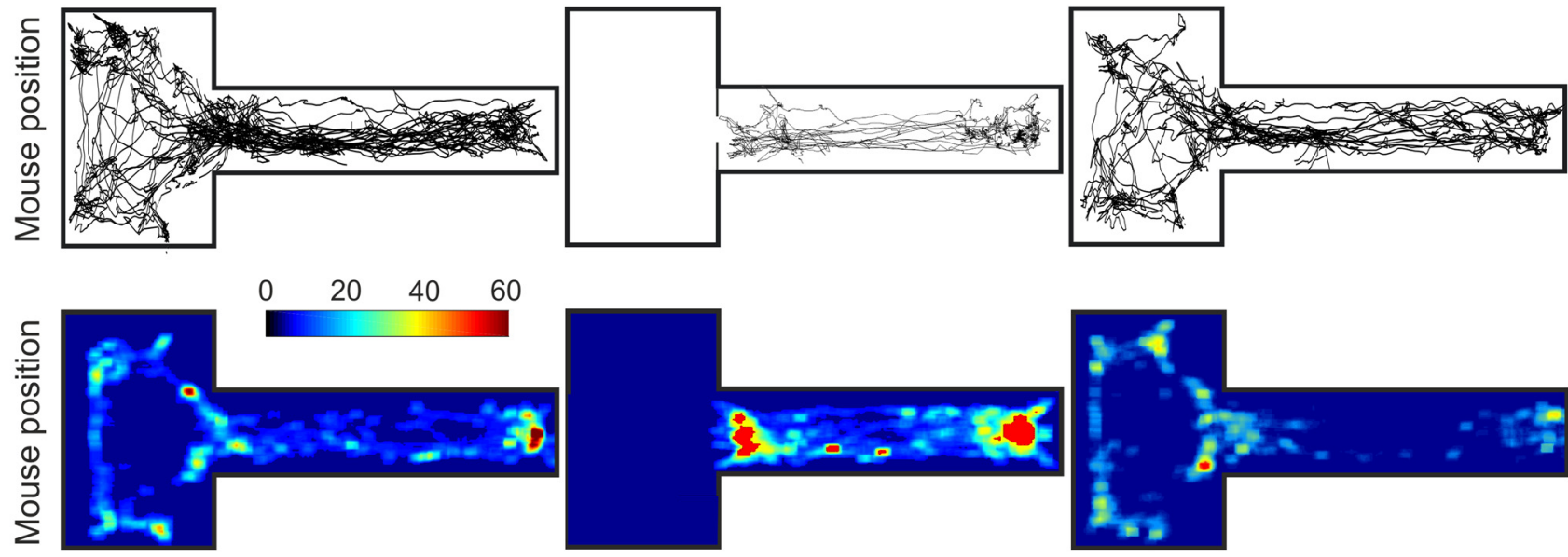

D

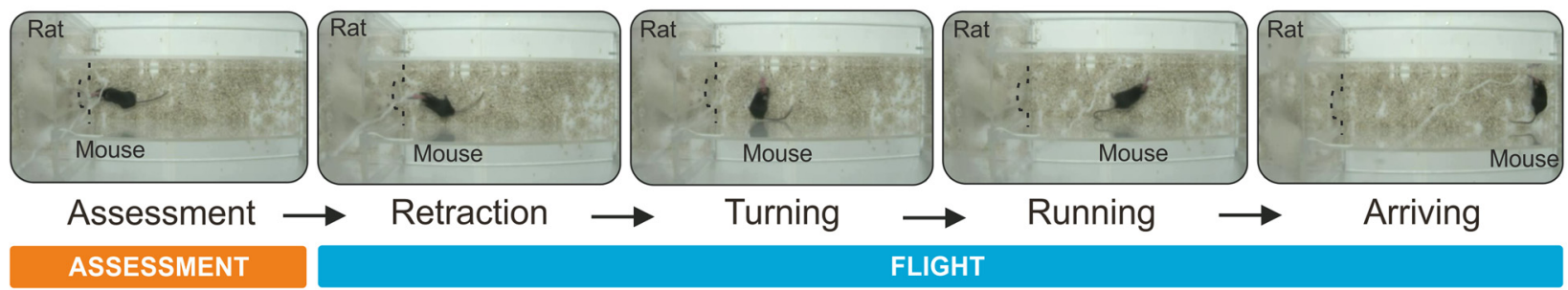

Figure 1. Predator exposure test apparatus. The mouse was transferred to the testing apparatus and allowed to explore the corridor and stimulus chamber first in the absence of the rat, then in the presence of the rat, and lastly without the rat. $\boldsymbol{A}$, Representative example of mouse position during pre-rat (left), rat (middle), and post-rat (right) phases. $\boldsymbol{B}$, Example of a representative center of mass mouse body tracking during the pre-rat (left), rat (middle), and post-rat (right) phases. $C$, Heat-map showing average cumulative time in the apparatus in the pre-rat (left), rat (middle), and post-rat (right) phases $(N=12)$. $\boldsymbol{D}$, Sequence of video frames for a representative mouse executing approach and flight during the rat exposure phase: approach (assessment) and flight (retraction, turning, running, arriving).

with both approach and flight behavior, but given that they were solitary units, we did not consider them a robust cell-type. Similar assessment + and flight + cells have been previously reported in dPAG (Deng et al., 2016). As assessment cells increased their firing rate during the approach toward the rat, we plotted their average firing rat against distance from the stimulus chamber. This analysis revealed a similar, nonlinear relationship between both assessment + and assessment- cell firing and distance (Fig. $2 F$ ) in which assessment cells moderated their firing only when close to the stimulus chamber. This relationship is consistent with the encoding of threat proximity by $\mathrm{dPAG}$ assessment cells. To understand whether flight cells in $\mathrm{dPAG}$ might encode motor features of flight we plotted the average firing rate of flight cells superimposed on the animal's velocity. This analysis revealed that peak flight cell firing occurred before peak velocity was attained (Fig. $2 G$ ) consistent with the encoding of a motor pattern initiator rather than a motor executor function for dPAG flight cells.

In VMHdm, $28 \%$ of single units (67/236) were significantly correlated with approach-flight behavior (Fig. $3 B$ ). The patterns of correlation could be clustered into three types - cells that increased firing during approach (assessment+ cells, 10\%,
24/236; Fig. 3C; Extended Data Fig. 2-2A; Movie 4), cells that increased firing during flight ("flight + " cells, 8\%, 19/236; Fig. $3 D$; Extended Data Fig. 2-2A; Movie 5), and cells that decreased firing during flight ("flight-" cells, 9\%, 21/236; Fig. 3E; Extended Data Fig. 2-2A; Movie 6). Three single units consistently decreased firing during approach, but given the small number of units with this pattern, we did not analyze this cell-type further. To examine what aspects of approach might be encoded by assessment + cells we plotted their average firing rate against distance from the stimulus chamber. This analysis revealed a linear relationship between assessment + cell firing and distance (Fig. $3 F)$ in which assessment + cells monotonically increased their firing rate as the animal approached the rat chamber. This relationship is consistent with the encoding of threat intensity by VMHdm assessment cells. To understand whether flight cells in VMHdm might encode motor features of flight we plotted the average firing rate of flight + cells superimposed on the animal's velocity. This analysis revealed that the peak firing of flight+ cells occurred shortly before peak velocity was attained (Fig. $3 G$ ). Using the initiation of flight as a common time point, we compared the profiles of firing rates of flight + cells in dPAG and VMHdm (Fig. 3H). Peak firing of dPAG flight + cells occurred 
A

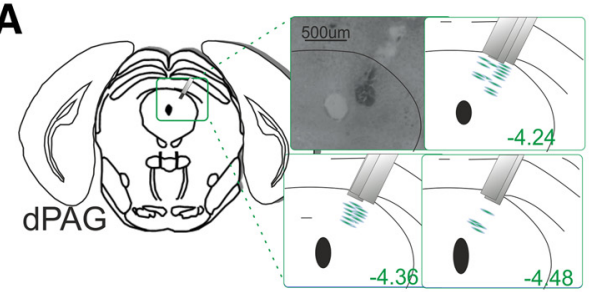

C
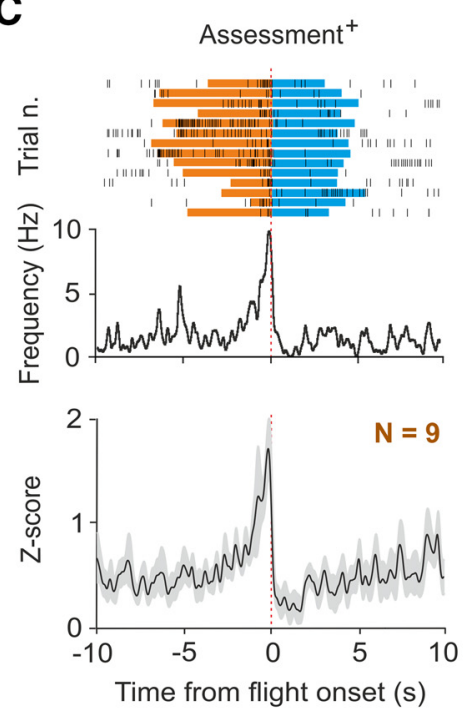

B

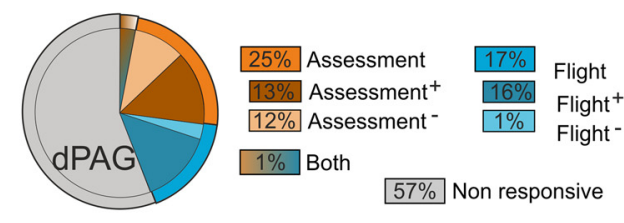

E
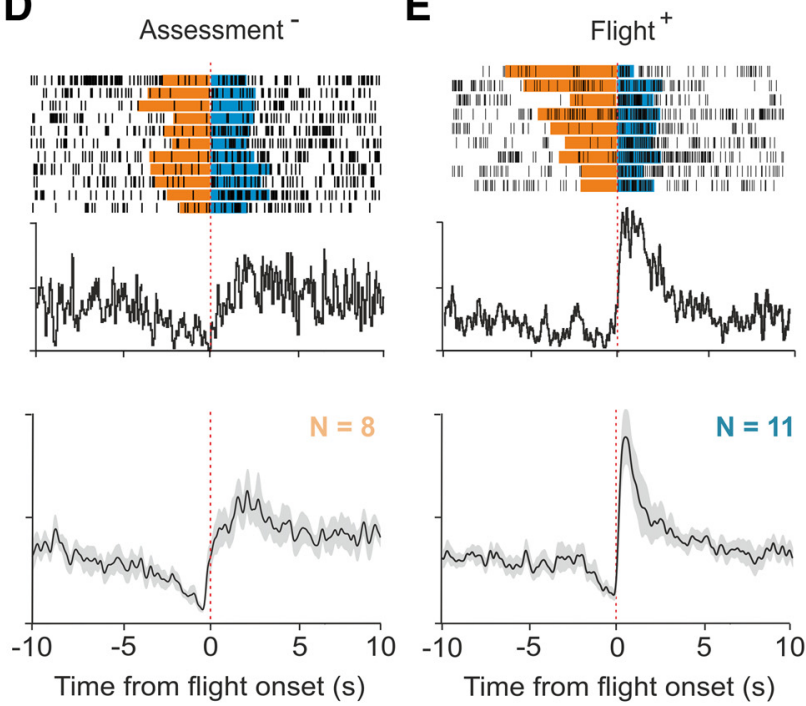

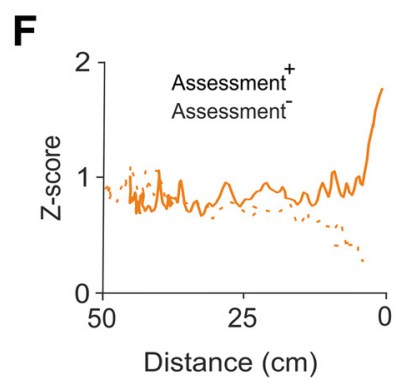

G

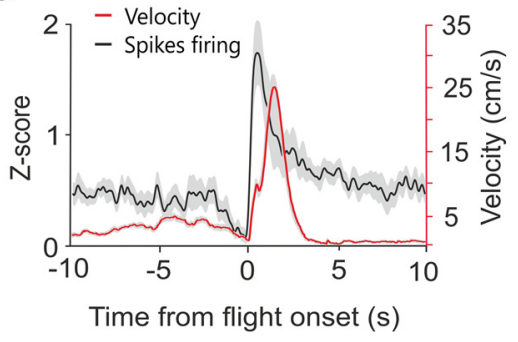

Figure 2. Single unit activity in dPAG during exposure to predator. $\boldsymbol{A}$, Nissl staining showing representative electrolytic lesion in dPAG and estimated electrode recording sites. $\boldsymbol{B}$, Population distribution of single units identified in dPAG (25\% assessment, $16 \%$ flight, $1 \%$ both; mice $N=8$, cells $N=69)$. Sorting examples can be found in Extended Data Figure 2-1. C, Firing frequency of a representative assessment+ cell over trials (top). Normalized average firing rate of all assessment+ cells identified (bottom, $N=9$ ). $D$, Firing frequency of a representative assessmentcell over trials (top). Normalized average firing rate of all assessment- cells identified (bottom, $N=8$ ). $\boldsymbol{E}$, Firing frequency of a representative flight + cell over trials (top). Normalized average firing rate of all flight + cells identified (bottom, $N=11$ ). $\boldsymbol{F}$, Normalized firing rates of assessment + (continuous line) and assessment- (dashed line) cells identified plotted against distance from the rat chamber. $\mathbf{G}$, Average firing activity of flight + cells and mouse velocity during flight. Time zero represents the flight onset. A summary of single unit firing rates and the average of single unit firing rates during different phases of predator exposure can be found in Extended Data Figures 2-2, 2-3, respectively.

on average $220 \mathrm{~ms}$ before that of VMHdm flight + cells. Consistent with an earlier peak firing in $\mathrm{dPAG}$ flight + cells, a comparison of the correlation between flight + cell firing and velocity revealed a steeper slope for dPAG compared with VMHdm (Fig. 3I). Although in the absence of data deriving from simultaneous recordings in the two structures, we cannot draw conclusions about the relative latency of neuronal recruitment, this comparison suggests that $\mathrm{dPAG}$ is more rapidly recruited during flight than VMHdm.

To determine whether the increase in firing rate exhibited by assessment cells as the animal approached the rat chamber was dependent on the presence of the rat, we analyzed single unit firing during the habituation phase. Although mice showed a similar number of approaches to the stimulus chamber during the habituation and rat phases (habituation 330, rat 334), firing rates of dPAG and VMHdm assessment cells did not increase during approach to the stimulus chamber in the absence of the rat (Extended Data Fig. 2-3). These findings demonstrate that assessment cell firing during approach toward the rat is dependent on predator sensory cues.

Next, we examined whether our finding that the peak firing of dPAG flight cells preceded that of VMHdm flight cells might depend on feedback from dPAG to VMHdm. Vglut2::Cre transgenic mice were implanted with a 16-microwire movable drive into the VMHdm and injected unilaterally in the dPAG with a mixture of viruses expressing channelrhodopsin (AAV5-Ef1a:: DIO-ChR2-EYFP) and hM4D (AAV2-hSyn::DIO-hM4D-Cherry) and implanted with an optic fiber above the ipsilateral dPAG (Fig. $4 A$ ). A total of 66 units in six mice were recorded in VMHdm while optogenetically stimulating and/or pharmacogenetically inhibiting Vglut2+ excitatory neurons in dPAG. This class of neurons is known to be capable of eliciting flight responses under 
A

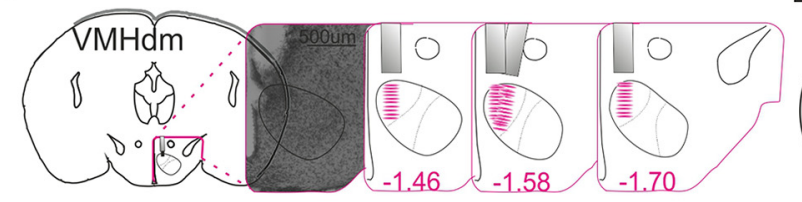

C
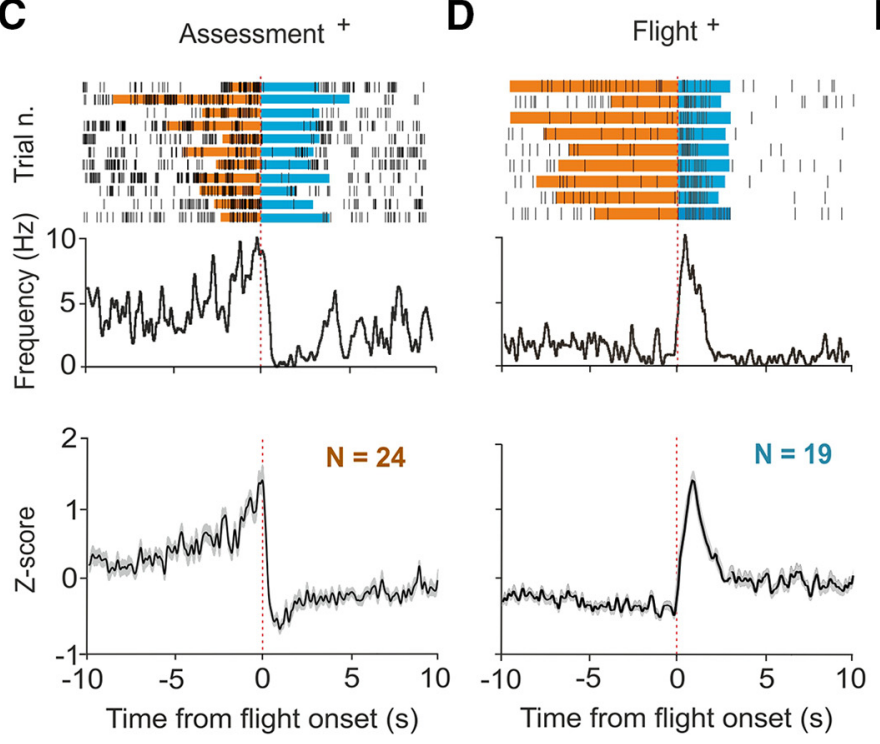

B

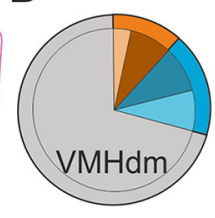

E

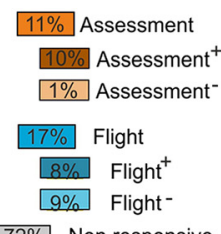

$72 \%$ Non responsive

Flight $^{-}$
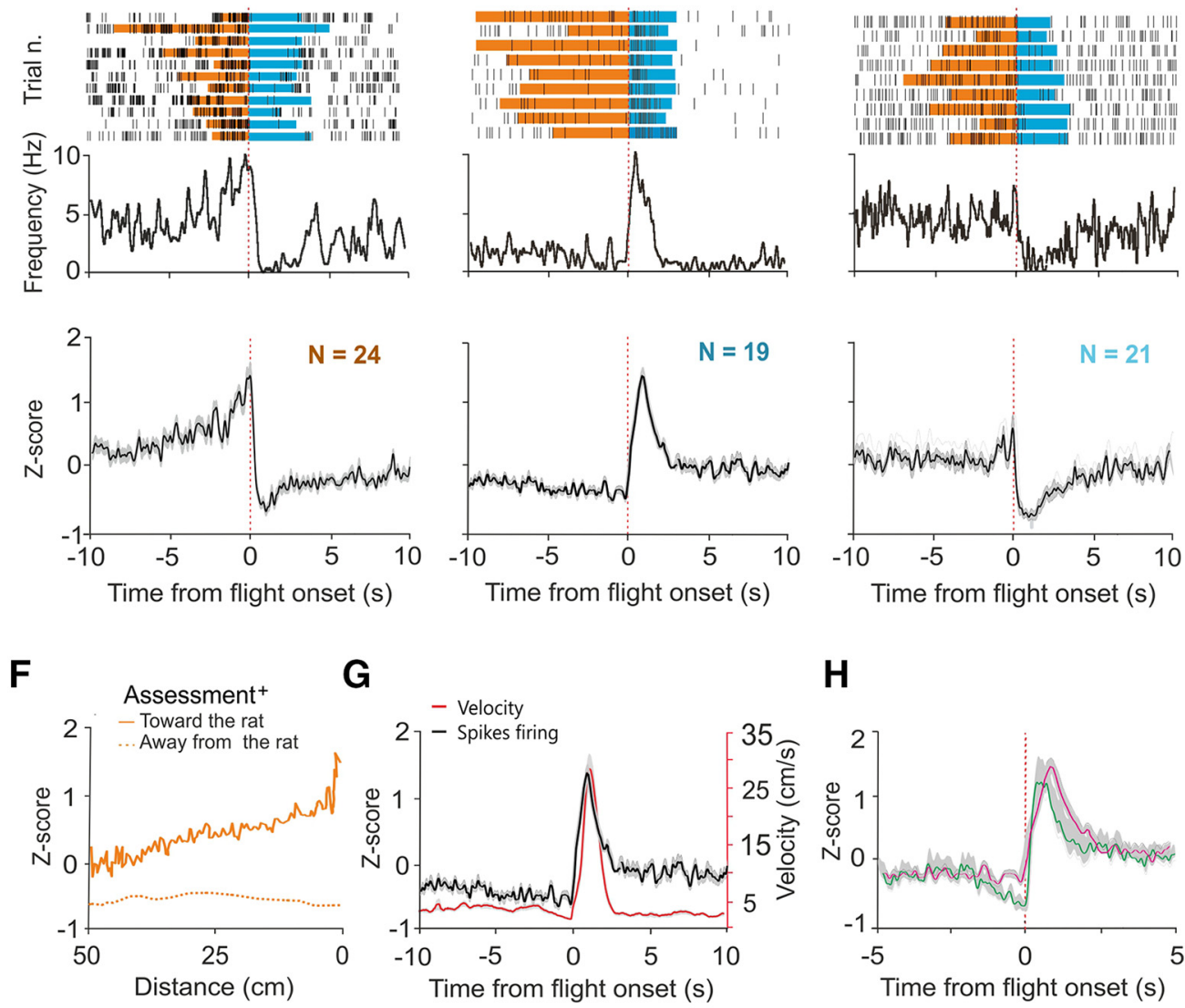

G

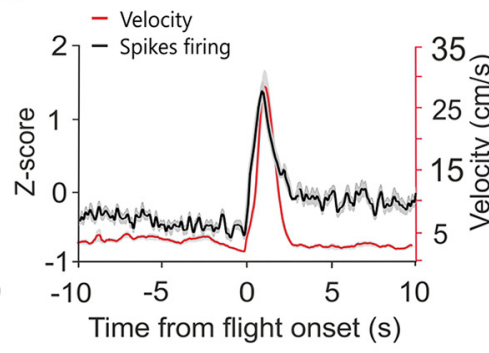

H
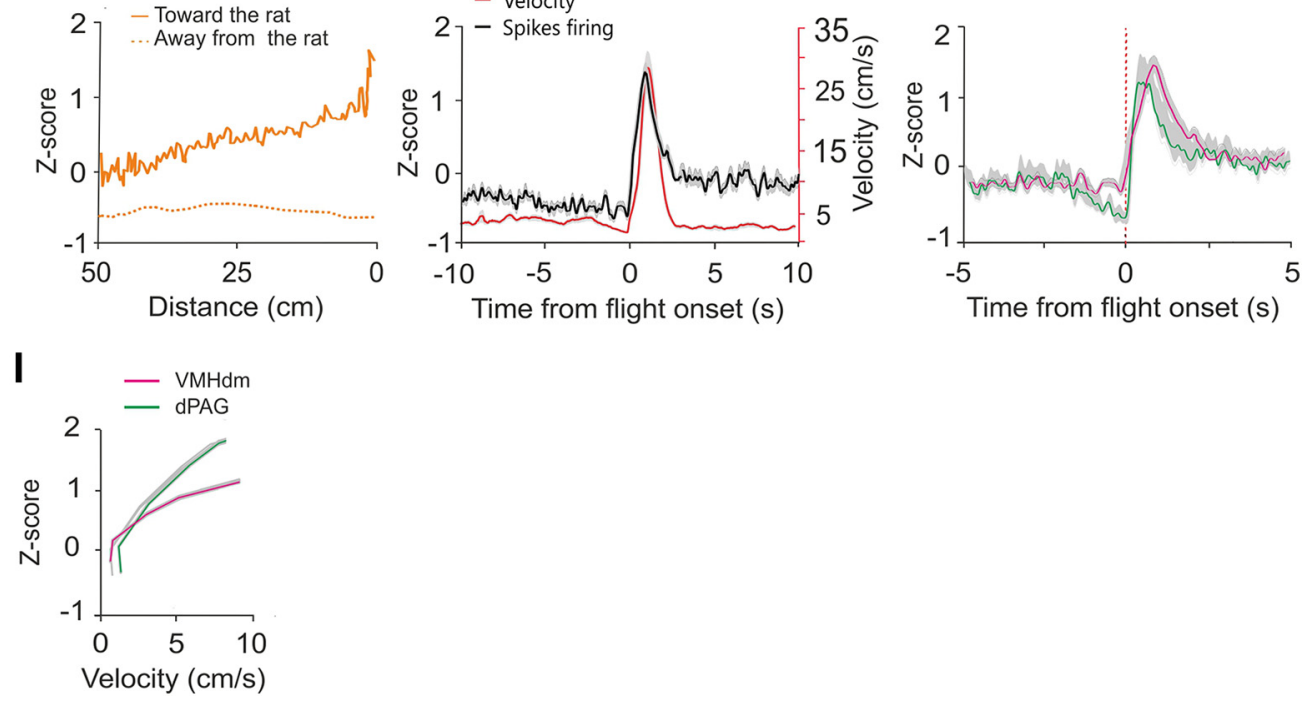

Figure 3. Single unit activity in VMHdm during exposure to predator. $\boldsymbol{A}$, Nissl staining showing representative electrolytic lesion in VMHdm and estimated electrode recording sites. $\boldsymbol{B}$, Population distribution of single units identified in VMHdm (11\% assessment, 17\% flight, mice $N=4$, cells $N=236)$. Sorting examples can be found in Extended Data Figure 3-1. C, Firing frequency of a representative assessment + cell over trials (top). Normalized average firing rate of all assessment + cells identified (bottom, $N=24)$. $\boldsymbol{D}$, Firing frequency of a representative flight + cell over trials (top). Normalized average firing rate of all flight + cells identified (bottom, $N=19$ ). $\boldsymbol{E}$, Firing frequency of a representative flight- cell over trials (top). Normalized average firing rate of all identified flight- cells (bottom, $N=21$ ). $\boldsymbol{F}$, Normalized firing rates of assessment + cells plotted against distance from the rat chamber. Continuous line indicates firing rate during approach and dashed line indicates firing rate during flight. $\boldsymbol{G}$, Average firing activity of flight + cells and mouse velocity during flight. $\boldsymbol{H}$, Normalized firing rate of flight + cells in dPAG (green) and VMHdm (magenta) during flight. I, Average firing activity of flight + cells in dPAG (green) and VMHdm (magenta) plotted against average velocity during flight. Time zero represents flight onset.

optogenetic stimulation (Deng et al., 2016; Tovote et al., 2016; Evans et al., 2018). In the initial phase of the experiment, animals were stimulated with light in the dPAG to activate excitatory neurons while recording VMHdm single units in their home cage. A large fraction of VMHdm units $(39 \%, 26 / 66)$ increased their firing activity when a train of light pulses was delivered to dPAG, while a smaller fraction $(15 \%, 10 / 66)$ decreased their firing activity (Figs. $3 C, 4 B)$. Excitatory and inhibitory response latencies were $15 \pm 3.1$ and $276 \pm 14 \mathrm{~ms}$, respectively (Fig. 4C). During the second phase of the experiment, animals were tested for rat approach 


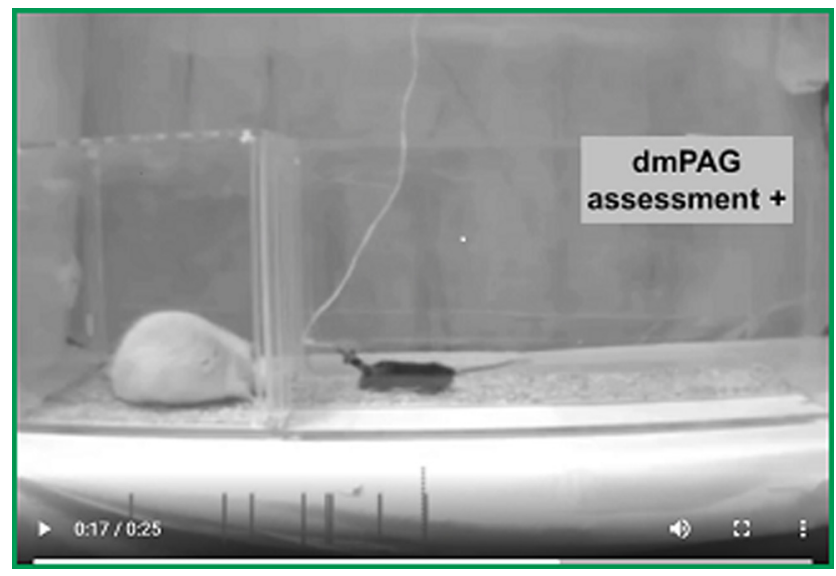

Movie 1. Representative video showing the activity of an assessment + cell recorded from dPAG. [View online]

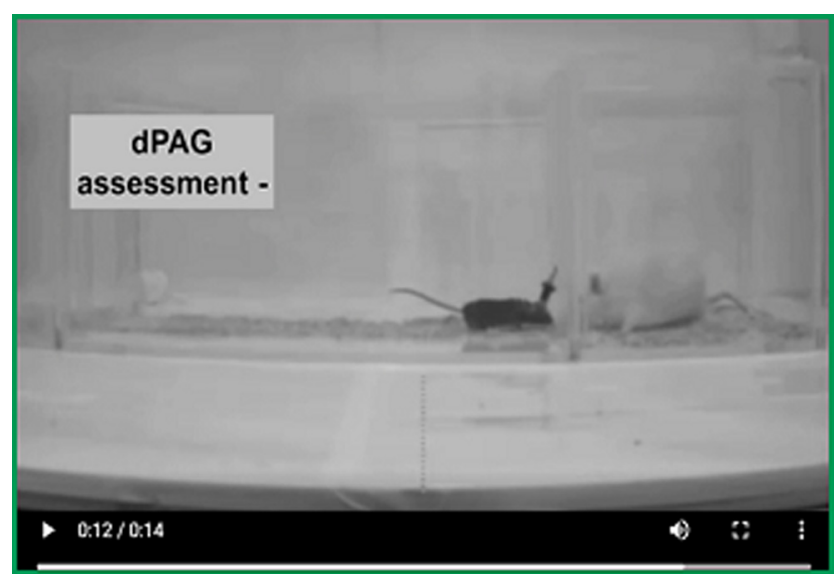

Movie 2. Representative video showing the activity of an assessment- cell recorded from dPAG. [View online]

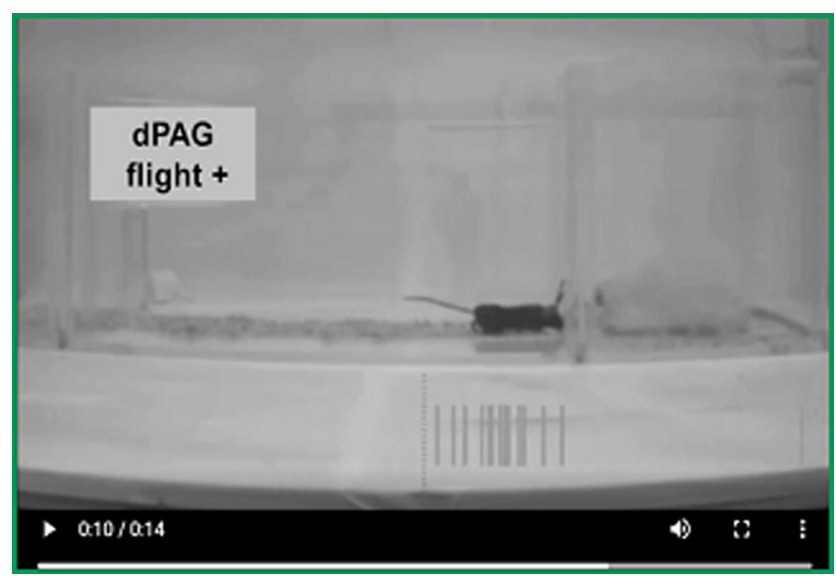

Movie 3. Representative video showing the activity of a flight + cell recorded from dPAG. [View online]

behavior as previously described and units were identified that significantly correlated with approach-flight behavior (15\%, 10/66; flight $+N=1$, flight $-N=5$, assessment $+N=3$, assessment$N=1$; Fig. 4B; Extended Data Fig. 4-1). Unexpectedly, flight + and assessment- cells consistently showed a decrease in firing rate

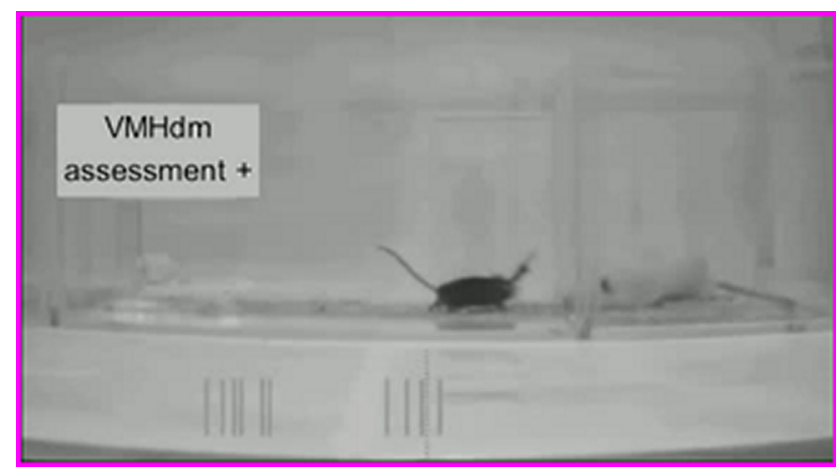

Movie 4. Representative video showing the activity of an assessment + cell recorded from VMHdm. [View online]

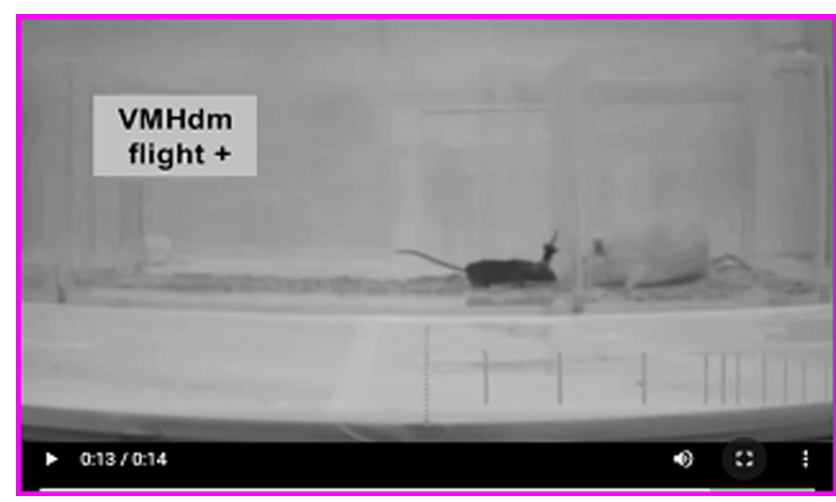

Movie 5. Representative video showing the activity of a flight + cell recorded from VMHdm. [View online]

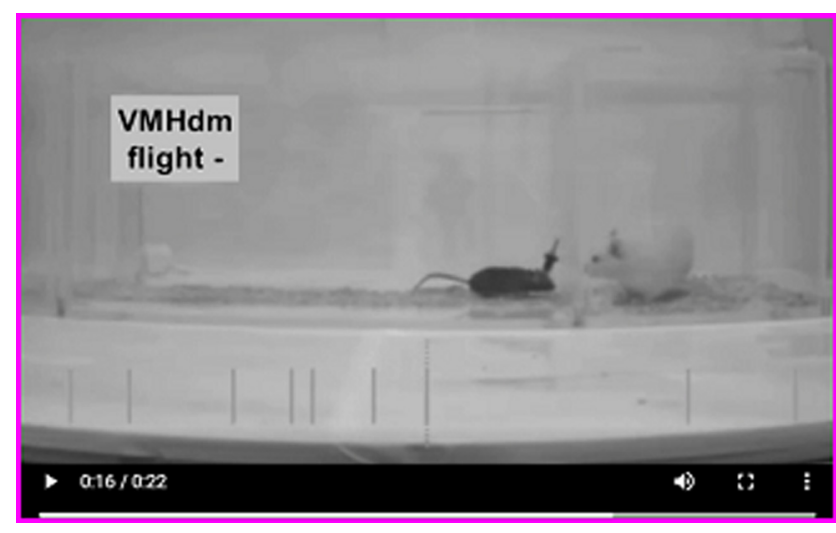

Movie 6. Representative video showing the activity of a flight- cell recorded from VMHdm. [View online]

during light stimulation, while flight- and assessment + cells consistently showed an increase in firing rate (Fig. 4D). These data suggest that neural activity in $\mathrm{dPAG}$ counteracts rather than promotes the rapid change in firing activity seen at the initiation of flight.

In the third phase of the experiment, mice were treated with the long-acting hM4D agonist CNO $(3 \mathrm{mg} / \mathrm{kg}$, i.p.) and tested again for ChR2-evoked responses. To confirm that hM4D activation successfully inhibited neurotransmission in excitatory dPAG neurons we compared light-evoked field potential (fEPSP) responses in VMHdm before and after $\mathrm{CNO}$ treatment. CNOtreated animals showed a significant decrease in the amplitude of 
the short latency evoked response (paired $t$ test; $p<0.001,95 \%$ confidence interval; Fig. $4 E$ ) consistent with a robust suppression of feedback from dPAG to VMHdm. When we compared dPAG light-evoked single unit activity in VMHdm before and after CNO treatment, we found, as expected, that assessment + and flight- cells showed a reduction in the amplitude of positive light-evoked responses. Unexpectedly, however, assessment- and flight + cells showed a switch from negative to positive light-evoked responses (Fig. 4F; Extended Data Fig. 4-1), suggesting that the partial inhibition of lightevoked neural activity in dPAG had unmasked a positive feedback circuit to these cells. Finally, in the last phase of the experiment, CNO-treated mice were tested for rat approach behavior as previously described, and the behavioral entrainment of VMHdm single units was compared before and after CNO treatment. Similar assessment-flight sequences were observed in this group of mice (after saline injection: $6.7 \pm 0.08$ per session; after $\mathrm{CNO}$ injection: $5.3 \pm 0.6 ; 4 \pm 1$ session per animal, maximum one session per day, $N=6$ ). Approach behavior was followed by flight during the rat phase (after saline injection: habituation $0 / 147$, rat $94 / 98$, postrat 3/64; after CNO injection; habituation 0/ 117 , rat $72 / 74$, post-rat $2 / 59$ ). In general, behavioral correlations of assessment + , assessment-, and flight- cells showed a small reduction in amplitude, while no change in flight + cell entrainment was detected. These data suggest that dPAG feedback plays a modulatory rather than driving role in VMHdm activity.

\section{Discussion}

We have recorded the firing of 371 putative single neurons in the mouse VMHdm and dPAG during exposure to a live rat. We found two classes of neurons among those that showed firing that was significantly correlated with the defensive behavioral responses of the experimental animals. One class showed firing whose rate was modulated as the animal approached the rat, called assessment cells, and the other whose rate was modulated as the animal fled the rat, called flight cells. Both classes of cells were found in VMHdm and dPAG, suggesting that both structures encode aspects of threat detection and avoidance consistent with the known requirement of these structures in the expression of predator defense.

However, several differences in the correlations between firing and behavior were evident in VMHdm and dPAG. For assessment + cells, firing rates increased in an inverse linear manner with distance from the rat in VMHdm, but in a delayed, nonlinear manner in dPAG (Figs. 2C, 3C). This difference suggests that VMHdm is activated earlier during approach to a predatory threat and that it directly encodes threat distance, while $\mathrm{dPAG}$ is activated only in close proximity to the predator as threat levels
B

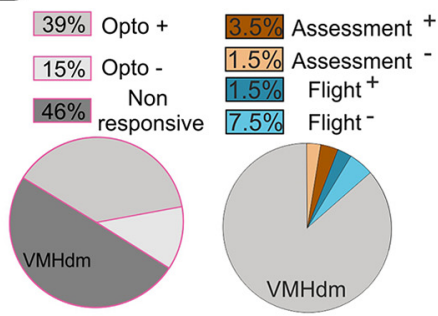

D assessment + - Flight +

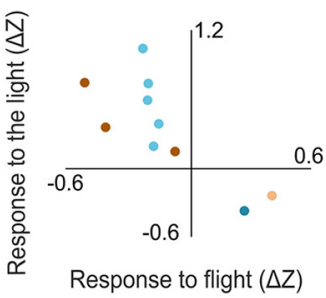

$\mathbf{F}$

- assessment + • Flight + assessment - Flight

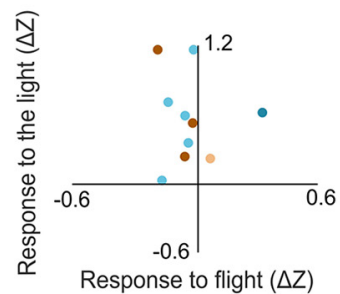

Figure 4. Manipulation of dPAG Glutamatergic cells and simultaneous recording in VMHdm cells. $A$, Schematic showtion of single units identified in VMHdm during optogenetic activation of dPAG (left, 39\% opto + and 15\% opto-, mice individual variation to pre-post light stimulation and pre-/post-flight episode before CNO injection. fl + and assessmentcells get both inhibited on ChR2 stimulation of Vglut2 + cells in the dPAG. fl- and assessment + cells get activated instead. $\boldsymbol{E}$, Comparison of the average amplitude of the field responses evoked in VMHdm by the first pulse of the optogenetic train stimulation in IPAG (top) before CNO injection (black traces) and after (red traces). The amplitude of the sessions $N=14$ ). $\boldsymbol{F}$, Same as $D$ after CNO injection. Traces of the activity of the cells during the exposure to rat before and after CNO injection can be found in Extended Data Figure 4-1. $* * * p \leq 0.001$.

rise beyond a certain threshold. Because VMHdm receives direct projections from the MeApv that encodes predator odor (Canteras et al., 1995; Choi et al., 2005; Bergan et al., 2014) it is reasonable to postulate that the increase in firing of assessment+ cells in VMHdm reflects their direct receipt of predator odor information from upstream olfactory and kairomone processing areas. However, VMHdm is also likely to receive non-olfactory sensory information about the predator from multimodal sensory processing areas of the amygdala, such as the basomedial and basolateral nucleus (Petrovich et al., 1996; Li et al., 2004; Martinez et al., 2011; Gross and Canteras, 2012). Given the highly linear relationship between VMHdm assessment + cell firing and predator distance, we argue that VMHdm assessment + cells are directly driven by multimodal sensory information about the threat. This hypothesis was confirmed by our observation that assessment cell firing during the habituation and post-rat phase was not increased as the animal approached the stimulus chamber in the absence of the rat (Extended Data Fig. 2-3). These data also demonstrate that 
predatory olfactory cues remaining in the stimulus chamber are not sufficient to recruit VMHdm or $\mathrm{dPAG}$ neuronal firing during the post-rat phase, a finding consistent with the failure to recruit cFos activity during predator contextual fear memory (Motta et al., 2009; Silva et al., 2016b), but seemingly at odds with the ability of pharmacogenetic inhibition of VMHdm to inhibit defensive behavior to the predator context (Silva et al., 2016b). However, methodological differences (e.g., housing, handling, and/or training-testing delay) may have reduced the recruitment of VMHdm during predator context recall in our study. Finally, we note that firing activity of $\mathrm{VMHvl}$ units during approach to conspecifics is also proportional to distance (Lin et al., 2011; Falkner et al., 2014), although in these studies the small size of the testing apparatus precluded an estimation of the linearity of this relationship.

Given that VMHdm neurons project heavily to dPAG and the hypothalamus provides over $45 \%$ of dPAG inputs (Beitz, 1982, 1989; Canteras et al., 1994; Silva et al., 2013; Wang et al., 2015), we speculate that the nonlinear relationship with predator distance shown by dPAG assessment cell firing (both assessment + and assessment- cells) rests in part on their being driven in a nonlinear manner by inputs from VMHdm. However, optogenetic stimulation of VMHdm-dPAG projections alone was able to elicit freezing, but not flight behavior (Wang et al., 2015), suggesting that other dPAG afferents may be required to reach the threshold for flight. Inputs from the PMD, for example, are required for flight responses to predator and these may provide the critical drive for dPAG flight cells to reach threshold (Canteras and Swanson, 1992; Blanchard et al., 2003). Although the olfactory and multimodal sensory processing areas of the amygdala do not project directly to dPAG (Canteras et al., 1995; Petrovich et al., 1996; Martinez et al., 2011), assessment cells in this structure could receive multimodal sensory information about the predator from thalamic and collicular inputs (Mantyh, 1982; Beitz, 1989; Vianna and Brandão, 2003; Schenberg et al., 2005; Silva et al., 2013). For example, a role for dPAG in thresholding cumulative threat information has been described for superior colliculus afferents (Evans et al., 2018).

One of the surprising findings of our study was the presence of flight cells in VMHdm. Their presence demonstrates that the medial hypothalamus encodes both sensory and motor information about defense. It remains to be determined which inputs drive VMHdm flight cells. One possibility is that they are driven by thresholding of excitatory inputs from local assessment+ cells. Another possibility, consistent with evidence for projections from PAG to VMH (Mantyh, 1983; Meller and Dennis, 1991; Cameron et al., 1995), is that flight cells in VMHdm are driven by feedback from flight cells in dPAG, although we cannot rule out that this feedback could also be polysynaptic. To test the presence of functional $\mathrm{dPAG}$ to VMHdm feedback we undertook gain and loss-of-function experiments in which we activated or inhibited Vglut2+ excitatory neurons in dPAG known to be sufficient to induce flight (Tovote et al., 2016; Evans et al., 2018) while recording from VMHdm. Unexpectedly, we found a consistent pattern of feedback in which cells that increased their firing at flight initiation (flight + , assessment-) were inhibited by dPAG feedback while cells that decreased their firing at flight initiation were activated (Fig. 4D). These data do not support the hypothesis that flight cells in $\mathrm{DPAG}$ are driving flight cells in VMHdm and instead argue the dPAG feedback counteracts the reciprocal changes in firing seen in VMHdm on flight onset, potentially dampening the propensity to induce flight. The inhibitory feedback seen in some cells on $\mathrm{dPAG}$ optogenetic stimulation also suggests the existence of prominent feedforward inhibition in VMHdm, a feature consistent with in vitro electrophysiological characterization of $\mathrm{VMH}$ afferents (Yamamoto et al., 2018). Interestingly, we observed a consistent switch in ChR2-evoked responses in these VMHdm cells from negative to positive when dPAG neurons were pharmacogenetically attenuated, suggesting that these cells may receive both feedforward inhibition and excitation from dPAG and that the former may be more evident at high activation levels. Additional experiments aimed at understanding the behavioral response profile of $\mathrm{dPAG}$ neurons providing feedback to VMHdm will be necessary to understand whether feedback in this system acts primarily to boost the activity of VMHdm assessment + cells as the animal approaches a threat and thus potentially promotes threat responses, or whether it acts to dampen the reciprocal switch in firing activity of VMHdm flight+ and assessment + cells that occurs at flight initiation to suppress escape responses.

\section{References}

Amano K, Tanikawa T, Kawamura H, Iseki H, Notani M, Kawabatake H, Shiwaku T, Suda T, Demura H, Kitamura K (1982) Endorphins and pain relief. Further observations on electrical stimulation of the lateral part of the periaqueductal gray matter during rostral mesencephalic reticulotomy for pain relief. Appl Neurophysiol 45:123-135.

Arendt D, Tosches MA, Marlow H (2016) From nerve net to nerve ring, nerve cord and brain - evolution of the nervous system. Nat Rev Neurosci 17:61-72.

Bandler R, McCulloch T (1984) Afferents to a midbrain periaqueductal grey region involved in the "defense reaction" in the cat as revealed by horseradish peroxidase. II. The diencephalon. Behav Brain Res 13:279-285.

Beitz AJ (1982) The organization of afferent projections to the midbrain periaqueductal gray of the rat. Neuroscience 7:133-159.

Beitz AJ (1989) Possible origin of glutamatergic projections to the midbrain periaqueductal gray and deep layer of the superior colliculus of the rat. Brain Res Bull 23:25-35.

Bergan JF, Ben-Shaul Y, Dulac C (2014) Sex-specific processing of social cues in the medial amygdala. Elife 3:e02743.

Blanchard DC, Williams G, Lee EMC, Blanchard RJ (1981) Taming of wild Rattus norvegicus by lesions of the mesencephalic central gray. Psychobiology 9:157-163.

Blanchard DC, Li CI, Hubbard D, Markham CM, Yang M, Takahashi LK, Blanchard RJ (2003) Dorsal premammillary nucleus differentially modulates defensive behaviors induced by different threat stimuli in rats. Neurosci Lett 345:145-148.

Borgius L, Restrepo CE, Leao RN, Saleh N, Kiehn O (2010) A transgenic mouse line for molecular genetic analysis of excitatory glutamatergic neurons. Mol Cell Neurosci 45:245-257.

Braak H, Braak E (1992) Anatomy of the human hypothalamus (chiasmatic and tuberal region). Prog Brain Res 93:3-14; discussion 14-16.

Cameron AA, Khan IA, Westlund KN, Cliffer KD, Willis WD (1995) The efferent projections of the periaqueductal gray in the rat: a Phaseolus vulgaris-leucoagglutinin study. I. Ascending projections. J Comp Neurol 351:568-584.

Canteras NS (2002) The medial hypothalamic defensive system: hodological organization and functional implications. Pharmacol Biochem Behav 71:481-491.

Canteras NS, Swanson LW (1992) The dorsal premammillary nucleus: an unusual component of the mammillary body. Proc Natl Acad Sci USA 89:10089-10093.

Canteras NS, Simerly RB, Swanson LW (1994) Organization of projections from the ventromedial nucleus of the hypothalamus: a Phaseolus vulgaris-leucoagglutinin study in the rat. J Comp Neurol 348:41-79.

Canteras NS, Simerly RB, Swanson LW (1995) Organization of projections from the medial nucleus of the amygdala: a PHAL study in the rat. J Comp Neurol 360:213-245.

Canteras NS, Chiavegatto S, Ribeiro do Valle LE, Swanson LW (1997) Severe reduction of rat defensive behavior to a predator by discrete hypothalamic chemical lesions. Brain Res Bull 44:297-305. 
Choi GB, Dong H-W, Murphy AJ, Valenzuela DM, Yancopoulos GD, Swanson LW, Anderson DJ (2005) Lhx6 delineates a pathway mediating innate reproductive behaviors from the amygdala to the hypothalamus. Neuron 46:647-660.

Deng H, Xiao X, Wang Z (2016) Periaqueductal gray neuronal activities underlie different aspects of defensive behaviors. J Neurosci 36:75807588.

Evans D, Vanessa A, Vale R, Ruehle S, Lefler Y, Branco T (2018) A synaptic threshold mechanism for computing escape decisions. Nature 558:590594.

Falkner AL, Dollar P, Perona P, Anderson DJ, Lin D (2014) Decoding ventromedial hypothalamic neural activity during male mouse aggression. J Neurosci 34:5971-5984.

Falkner AL, Grosenick L, Davidson TJ, Deisseroth K, Lin D (2016) Hypothalamic control of male aggression-seeking behavior. Nat Neurosci 19:596-604

Fuchs SA, Edinger HM, Siegel A (1985) The organization of the hypothalamic pathways mediating affective defense behavior in the cat. Brain Res 330:77-92.

Graeff FG (1994) Neuroanatomy and neurotransmitter regulation of defensive behaviors and related emotions in mammals. Braz J Med Biol Res 27:811-829.

Gross CT, Canteras NS (2012) The many paths to fear. Nat Rev Neurosci 13:651-658.

Koutcherov Y, Mai JK, Ashwell KWS, Paxinos G (2002) Organization of human hypothalamus in fetal development. J Comp Neurol 446:301-324.

Kruk MR, van der Poel AM, de Vos-Frerichs TP (1979) The induction of aggressive behaviour by electrical stimulation in the hypothalamus of male rats. Behaviour 70:292-322.

Krzywkowski P, Penna B, Gross CT (2020) Dynamic encoding of social threat and spatial context in the hypothalamus. Elife 9:e57148.

Kunwar PS, Zelikowsky M, Remedios R, Cai H, Yilmaz M, Meister M, Anderson DJ (2015) Ventromedial hypothalamic neurons control a defensive emotion state. Elife 4:e06633.

Kurrasch DM, Cheung CC, Lee FY, Tran PV, Hata K, Ingraham HA (2007) The neonatal ventromedial hypothalamus transcriptome reveals novel markers with spatially distinct patterning. J Neurosci 27:13624-13634.

Li CI, Maglinao TL, Takahashi LK (2004) Medial amygdala modulation of predator odor-induced unconditioned fear in the rat. Behav Neurosci 118:324-332.

Lin D, Boyle MP, Dollar P, Lee H, Lein ES, Perona P, Anderson DJ (2011) Functional identification of an aggression locus in the mouse hypothalamus. Nature 470:221-226.

Malsbury CW, Kow L-M, Pfaff DW (1977) Effects of medial hypothalamic lesions on the lordosis response and other behaviors in female golden hamsters. Physiol Behav 19:223-237.

Mantyh PW (1982) Forebrain projections to the periaqueductal gray in the monkey, with observations in the cat and rat. J Comp Neurol 206:146158.

Mantyh PW (1983) Connections of midbrain periaqueductal gray in the monkey. I. Ascending efferent projections. J Neurophysiol 49:567-581.

Martinez RC, Carvalho-Netto EF, Ribeiro-Barbosa ER, Baldo MVC, Canteras NS (2011) Amygdalar roles during exposure to a live predator and to a predator-associated context. Neuroscience 172:314-328.

Meller ST, Dennis BJ (1991) Efferent projections of the periaqueductal gray in the rabbit. Neuroscience 40:191-216.

Motta SC, Goto M, Gouveia FV, Baldo MVC, Canteras NS, Swanson LW (2009) Dissecting the brain's fear system reveals the hypothalamus is critical for responding in subordinate conspecific intruders. Proc Natl Acad Sci USA 106:4870-4875.

Nomoto K, Lima SQ (2015) Enhanced Male-Evoked Responses in the ventromedial hypothalamus of sexually receptive female mice. Curr Biol 25:589-594.

Petrovich GD, Risold PY, Swanson LW (1996) Organization of projections from the basomedial nucleus of the amygdala: a PHAL study in the rat. J Comp Neurol 374:387-420.

Pfaff DW, Sakuma Y (1979a) Facilitation of the lordosis reflex of female rats from the ventromedial nucleus of the hypothalamus. J Physiol 288:189202.

Pfaff DW, Sakuma Y (1979b) Deficit in the lordosis reflex of female rats caused by lesions in the ventromedial nucleus of the hypothalamus. J Physiol 288:203-210.

Remedios R, Kennedy A, Zelikowsky M, Grewe BF, Schnitzer MJ, Anderson DJ (2017) Social behaviour shapes hypothalamic neural ensemble representations of conspecific sex. Nature 550:388-392.

Schenberg LC, Póvoa RMF, Costa ALP, Caldellas AV, Tufik S, Bittencourt AS (2005) Functional specializations within the tectum defense systems of the rat. Neurosci Biobehav Rev 29:1279-1298.

Silva BA, Mattucci C, Krzywkowski P, Murana E, Illarionova A, Grinevich V, Canteras NS, Ragozzino D, Gross CT (2013) Independent hypothalamic circuits for social and predator fear. Nat Neurosci 16:1731-1733.

Silva BA, Gross CT, Gräff J (2016a) The neural circuits of innate fear: detection, integration, action, and memorization. Learn Mem 23:544-555.

Silva BA, Mattucci C, Krzywkowski P, Cuozzo R, Carbonari L, Gross CT (2016b) The ventromedial hypothalamus mediates predator fear memory. Eur J Neurosci 43:1431-1439.

Stüttgen M (2020) MLIB - toolbox for analyzing spike data. MATLAB Central File Exchange. Available from https://www.mathworks.com/ matlabcentral/fileexchange/37339-mlib-toolbox-for-analyzing-spikedata.

Swanson LW (2000) Cerebral hemisphere regulation of motivated behavior. Brain Res 886:113-164.

Swanson LW, Petrovich GD (1998) What is the amygdala? Trends Neurosci 21:323-331.

Tessmar-Raible K, Raible F, Christodoulou F, Guy K, Rembold M, Hausen H, Arendt D (2007) Conserved sensory-neurosecretory cell types in annelid and fish forebrain: insights into hypothalamus evolution. Cell 129:1389-1400.

Tovote P, Esposito MS, Botta P, Chaudun F, Fadok JP, Markovic M, Wolff SBE, Ramakrishnan C, Fenno L, Deisseroth K, Herry C, Arber S, Lüthi A (2016) Midbrain circuits for defensive behaviour. Nature 534:206-212.

Vianna DML, Brandão ML (2003) Anatomical connections of the periaqueductal gray: specific neural substrates for different kinds of fear. Braz J Med Biol Res 36:557-566.

Wang L, Chen IZ, Lin D (2015) Collateral pathways from the ventromedial hypothalamus mediate defensive behaviors. Neuron 85:1344-1358.

Wilent WB, Oh MY, Buetefisch CM, Bailes JE, Cantella D, Angle C, Whiting DM (2010) Induction of panic attack by stimulation of the ventromedial hypothalamus. J Neurosurg 112:1295-1298

Yamamoto R, Ahmed N, Ito T, Zeynep Gungor N, Pare D (2018) Optogenetic study of anterior BNST and basomedial amygdala projections to the ventromedial hypothalamus. eNeuro 5: ENEURO.020418.2018.

Yang CF, Chiang MC, Gray DC, Prabhakaran M, Alvarado M, Juntti SA, Unger EK, Wells JA, Shah NM (2013) Sexually dimorphic neurons in the ventromedial hypothalamus govern mating in both sexes and aggression in males. Cell 153:896-909. 